\title{
10 years' experience with regular haemodialysis and renal transplantation
}

\author{
C CHANTLER, J E CARTER, M BEWICK, R COUNAHAN, J S CAMERON, C S OGG, \\ D G WILLIAMS, AND E WINDER
}

Evelina Children's Department, Guy's Hospital, London

SUMMARY 75 children aged under 15 years have entered the regular haemodialysis/renal transplant programme at Guy's Hospital in the 10 years since its inception; 13 children have subsequently died. A combination of hospital and home haemodialysis and renal transplantation was used. 64 children received 80 renal allografts; 37 1st grafts were from live, related donors and 27 were from cadaver donors. The 5-year acturial patient survival for the whole group and for those who had transplants was $76 \%$. Live donor graft survival was $65 \%$ at 3 years, and $55 \%$ at 5 years; 1 st cadaver graft survival was $42 \%$ at 3 years. Results obtained during the last 6 years of the 10-year period showed an improvement with a live related donor graft survival rate of $71 \%$, and a 1 st cadaver graft survival rate of $47 \%$ at 3 years. Particular experience was gained with home haemodialysis and live, related donor transplantation. Despite growth, and psychosocial and rehabilitation problems, the overall results were encouraging, particularly for the 46 children who had successful transplants. These children grew better, had fewer psychosocial difficulties, and were rehabilitated more successfully into normal life than those on long-term dialysis. We conclude that dialysis and transplantation should be offered to all suitable children with terminal renal failure.

The most recent data from the Paediatric Registry of the European Dialysis and Transplantation Association suggests that each year about 1.5 children per million population under age 15 years require dialysis and transplantation in order to survive. ${ }^{1}$ Just over a decade ago the outlook for such children was bleak, with many people suggesting that dialysis and transplantation should not be offered in view of the poor results and the heavy strain on family and child. ${ }^{2}$ Despite this, many centres undertook longterm dialysis and transplantation for children.

The first renal transplant was performed at Guy's Hospital in May $1967 .^{3}$ The first child was accepted into the regular haemodialysis programme in September 1968, and the early results of treatment have been reported. ${ }^{4}$ The joint experience of the Royal Free Hospital and Guy's Hospital with home

Evelina Children's Department, Guy's Hospital C CHANTLER, consultant paediatric nephrologist J E CARTER, honorary senior registrar

M BEWICK, consultant transplant surgeon

$R$ COUNAHAN, senior registrar

J S CAMERON, professor of renal medicine

C s OGG, consultant nephrologist

D G WILLIAMS, consultant nephrologist

E WINDER, nursing officer haemodialysis in children has been described. ${ }^{5}$ The results are now given of dialysis and transplantation in all children entered into the programme up to December 1978 at Guy's Hospital. The number of children each year is shown in Fig. 1 .

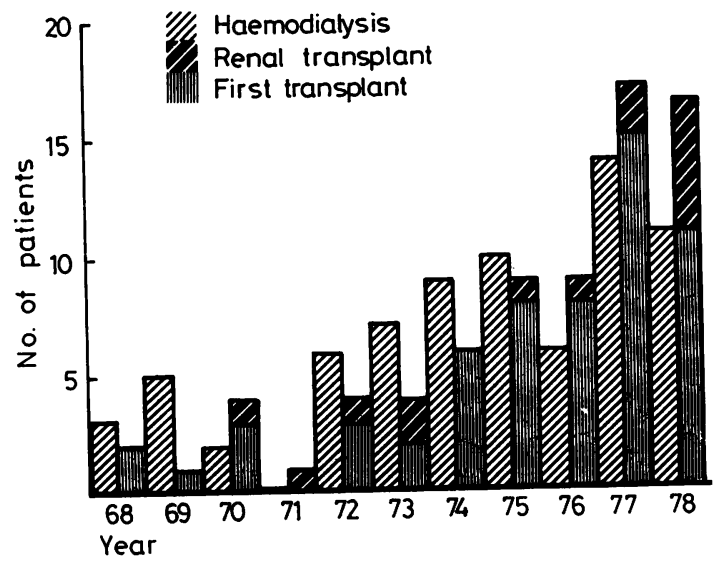

Fig. 1 Number of children accepted for haemodialysis each year, number of 1st transplants, and the total transplants each year. 


\section{Patients}

75 children who were under age 15 years at the time they developed end-stage renal failure were treated. Children over age 15 are excluded from this report, despite the fact that they were cared for by the paediatric staff. The youngest child accepted for regular haemodialysis was $4 \cdot 2$ years; the youngest child to be given a transplant was $6 \cdot 2$ years. The ages of the children at the start of treatment are shown in Fig. 2.

20 patients received peritoneal dialysis as an initial form of treatment. Each had presented in acute renal failure or acute on chronic renal failure, or had such severe metabolic abnormalities that dialysis was urgently required. One child with haemolytic uraemic syndrome was maintained on longterm peritoneal dialysis at home for 14 months before haemodialysis and transplantation.

72 children started hospital haemodialysis which was continued for between 1 and 6 months; 26 of them progressed to home haemodialysis. In addition, 2 other children went on to home haemodialysis after renal transplants had failed and one returned to home haemodialysis after a second attempt at renal transplantation.

64 of the children received 80 renal allografts from 37 live related and 43 cadaver donors. Three patients received their 1st renal transplants as the initial form of treatment. The flow of all these patients is shown in Fig. 3. Fewer children have been placed on home haemodialysis recently (Fig. 4), reflecting the preference for transplantation.

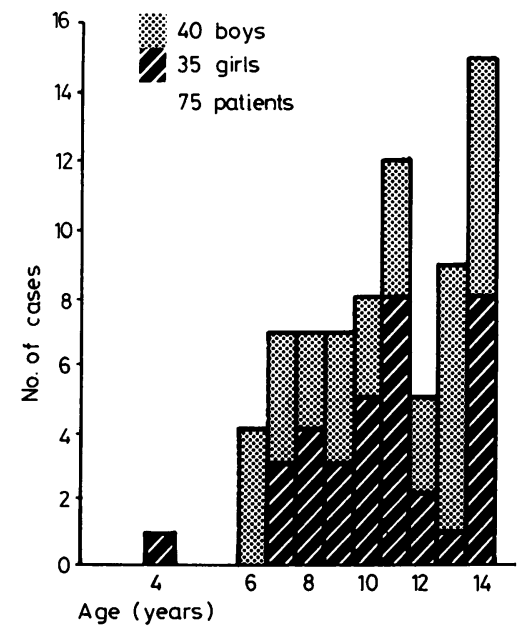

Fig. 2 Number and sex of children according to age at start of treatment.

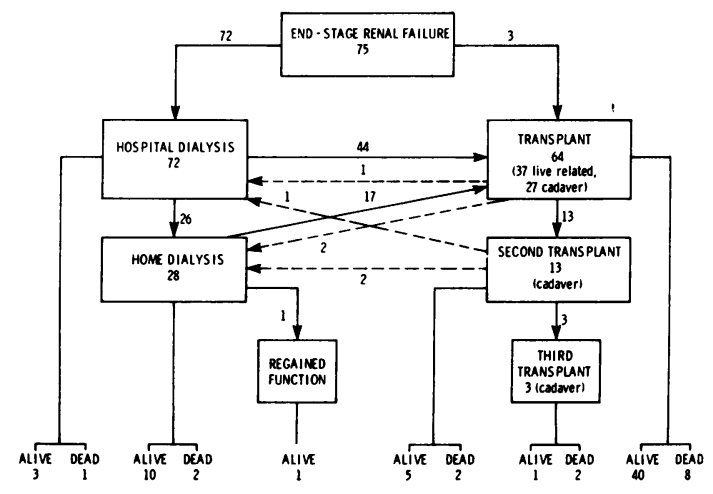

Fig. 3 Flow of patients between different modes of treatment and final status according to last mode of treatment in June 1978.

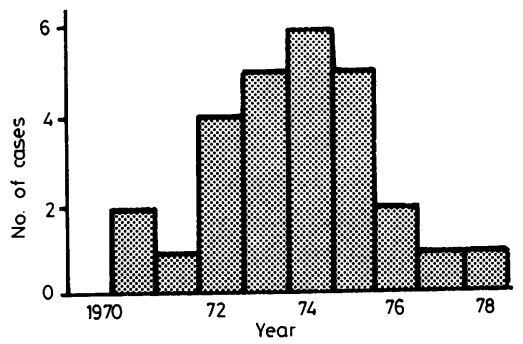

Fig. 4 New cases established on home haemodialysis each year.

Criteria for acceptability. The acceptance of a child with end-stage renal failure may be limited by the lack of a dialysis machine or, more often, lack of trained personnel; in 19782 children were refused for these reasons. Age and size of the child were important criteria; the youngest child to be haemodialysed on the regular hospital programme was $4 \cdot 2$ years and weighed $17 \cdot 3 \mathrm{~kg}$, and the youngest child to be given a transplant was 6.2 years and weighed $13 \mathrm{~kg}$. Younger, smaller children (including infants of $3.5 \mathrm{~kg}$ ) however, have been haemodialysed for short periods for acute renal failure.

Treatment was generally not advised in the presence of severe mental retardation or severe physical disabilities-such as blindness, congenital deafness, or paraplegia including spina bifida. One social reason for refusal was that the child came from a large family which was already under severe stress; another reason was if one parent, or both of them, had grave doubts about treatment. If treatment was not advised for reasons other than lack of resources in each case the parents were told that although it was against our judgement we would accept the child 
if they insisted; in no instance did this happen. In the joint Royal Free Hospital/ Guy's Hospital home dialysis study ${ }^{5} 19$ of 45 children were not accepted in the 2-year period 1973-74. Since then, greater experience has encouraged a more liberal policy so that slight mental retardation, psychiatric or behavioural disorder, or psychological or family disruption is not now a contraindication although it is recognised that each can add immensely to the care of such families. In the early part of the study, screening for referral was usually made by paediatricians and practitioners locally, whereas now our advice is more often sought before the decision is made and the child is seen by us either in the referring hospital or at Guy's. The increasing number of patients shown in Fig. 1 reflects these factors.

Apart from excluding any child with oxalosis the possibility of recurrence of the original disease in the transplanted kidney was not considered in the decision regarding transplantation in this series.

Origin of patients. Guy's Hospital is administered by the South-east Thames Regional Health Authority and serves the south-east area of England. Fig. 5 shows the distribution of patients we treated; no child from abroad was accepted for cadaver transplantation, nor, if facilities were inadequate at the time, was a child from abroad accepted for treatment. With the exception of 19 children, the remainder of the UK patients came from places outside the Greater London area and travelled up to 120 miles to the hospital; technical services for the home dialysis of these patients were provided from the unit.

Primary renal disease. The primary renal disease is shown in Fig. 6. The major cause of end-stage renal failure in children was pyelonephritis, with or without demonstrable vesicoureteric reflux (16 patients). Congenital abnormalities, including dyplastic or hypoplastic kidneys, were seen in 11

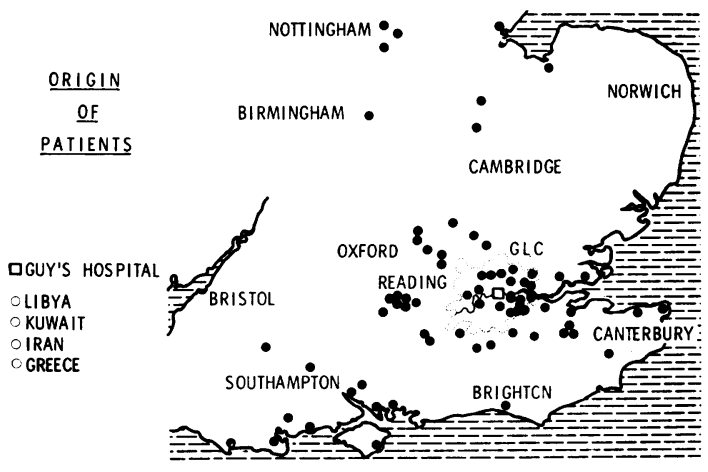

Fig. 5 Origin of children accepted for treatment.

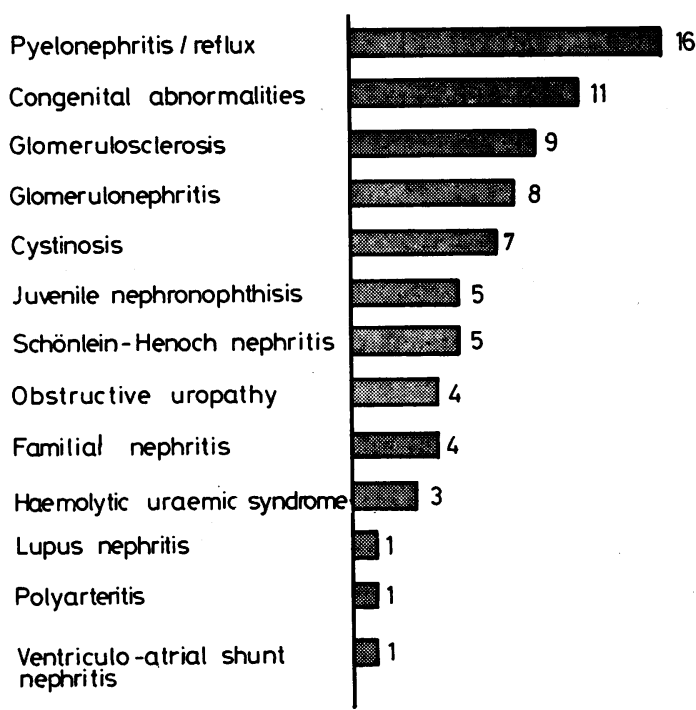

Fig. 6 Primary renal disease.

patients. Focal glomerulosclerosis and glomerulonephritis were the next most common. There were 7 patients with cystinosis.

\section{Methods}

The surgical techniques have been fully described elsewhere. ${ }^{6}$

Haemodialysis. Vascular access was by standard Quinton-Scribner Silastic arterio-venous shunts with Teflon tips. Shunts preferably in the leg were used for short-term dialysis before the creation of an arteriovenous fistula, and for patients admitted for live donor transplants. The posterior tibial artery or long saphenous vein just above the ankle was used most often but, in small patients, the brachial artery or the femoral artery was used. Subcutaneous Brescia-Cimino type fistulae, unilateral or bilateral, were generally created in the forearm several months before dialysis was necessary.

Children in hospital were dialysed three times a week for between 6 and 8 hours each dialysis; appropriately-sized flat plate disposable dialysers, mainly from the Gambro Lundia range, were used once only. ${ }^{7}$ In the home, dialysis usually took place three times a week for $\mathbf{8}$ hours overnight using nondisposable Meltec multipoint dialysers according to size; these were reused two or three times and rebuilt each week. The domestic water supply was softened before use but, except in the case of one child who developed a haemolytic anaemia due to chloramine sensitivity, ${ }^{8}$ deionisers were not used. 
Later in the study shorter dialysis (up to 5 hours three or four times a week in the evening after school) was introduced because better school attendance could be obtained. ${ }^{5}$ All the children at some time had $x$-ray evidence of secondary hyperparathyroidism or osteomalacia, or both, and received calciferol or, later, dihydrotachysterol for varying periods to control the bone disease. A free diet was encouraged, apart from sodium and water restriction. A dietary record was completed every month for a 3-day period $^{9}$ and was analysed by computer, and specific dietary adjustments were undertaken by the dietitian and child together. If energy or protein intake was inadequate supplements were used. ${ }^{10}$ Hyperphosphataemia was controlled by dietary adjustments or by consuming aluminium hydroxide preparations with meals. Each child was reviewed by the team of doctor, nurse, dietitian, and social worker at roughly monthly intervals. $X$-rays of the left hand and wrist were taken every 3 months for bone age and to assess osteodystrophy; pubertal staging was undertaken every 6 months. Children not previously immunised are now given measles vaccine at presentation.

\section{Renal transplantation.}

\section{Surgical management}

Most allografts were transplanted retroperitoneally into the iliac fossa and the renal artery anastomosed to the aorta or common iliac artery The renal vein was anastomosed end to side to the inferior vena cava or common iliac vein. The ureter in most cases was implanted using the Leadbetter-Politano technique.

Bilateral nephrectomy before transplantation was performed in 16 children because of upper tract dilatation or vesicoureteric reflux; while in 2 children hypertension after transplantation required nephrectomy. Only one graft into an ileal loop was undertaken; ureters were successfully placed in the bladders in all the other children who had had obstructive uropathy as the primary renal disease. HLA matching and cytotoxic cross-matching were carried out for all grafts, and mixed lymphocyte cultures for some. All children had received blood transfusions before transplants but no formal policy to transfuse patients before transplantation was undertaken for fear of sensitising patients.

\section{Medical management}

$1 \mathrm{~g}$ methyl-prednisolone and $120 \mathrm{mg} / \mathrm{m}^{2}$ azathioprine were given during the operation. Thereafier medical management, particularly of the latest cases, consisted of prednisolone $60 \mathrm{mg} / \mathrm{m}^{2}$ twice daily tapered rapidly over about 30 weeks to $20 \mathrm{mg} / \mathrm{m}^{2}$ given on alternate days, plus azathioprine $120 \mathrm{mg} / \mathrm{m}^{2}$ reduced to $60 \mathrm{mg} / \mathrm{m}^{2}$ after 2 weeks and maintained thereafter at this level unless an adjustment had to be made for leucopenia, infection, or hepatic dysfunction. Daily clinical assessment with monitoring of weight, blood pressure, plasma electrolytes, creatinine, and blood count continued for 1 month and the frequency was then gradually reduced, depending on progress, to weekly by 4-6 months, then monthly by 12-18 months after transplant. Rejection was diagnosed by a rise in the level of plasma creatinine, other clinical findings, or from the result of dynamic renal scintigraphy which was performed frequently starting immediately after the operation; the techniques and results have been discussed in more detail elsewhere. ${ }^{11}$ Percutaneous renal biopsy was undertaken if there was any doubt about the presence of rejection. Rejection episodes were treated with daily injections of methylprednisolone, $30 \mathrm{mg} / \mathrm{kg}$, for 3 days and, until recently, with intravenous actinomycin and radiotherapy to the graft daily for $\mathbf{3}$ days up to a maximum of three courses in all.

\section{Results}

Patient survival in all 75 children with end-stage renal failure was $76 \%$ at 5 years, and the 5 -year survival rate of the 57 children treated in the last 6 years was $83 \%$ (Fig. 7).

\section{Dialysis.}

\section{Patient survival}

Currently 3 patients are on hospital haemodialysis and 10 patients are on home haemodialysis. The 10 patients have been on home haemodialysis from periods of between 18 and 120 months. Actuarial 5 -year survival on dialysis was $94 \%$ at 5 years (Fig. 7). One patient on hospital haemodialysis for 7 months remained too sick to be transferred to home dialysis and she eventually died after an episode of

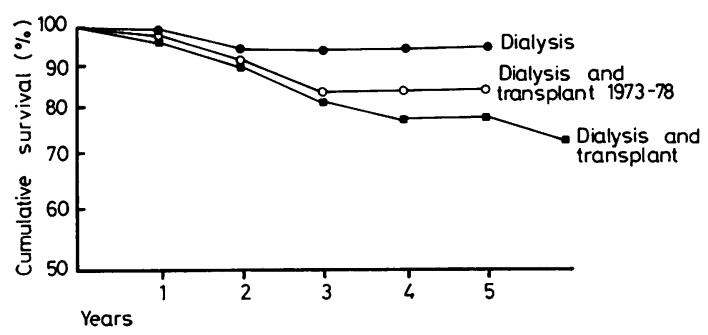

Fig. 7 Actuarial cumulative survival of patients $(<15$ years) on dialysis alone and those dialysed and transplanted. 
hypotensive collapse and convulsions associated with hypokalaemia. Two patients on home dialysis died, one after peptic ulceration and sepsis, and the other after septicaemia.

\section{Complications}

All the children were anaemic and were transfused, generally with washed red cells when the haemoglobin concentration fell below $5 \mathrm{~g} / \mathrm{dl} ; 3$ children had splenectomies associated with a rise in haemoglobin after red cell survival studies had indicated hypersplenism. A mean of 2 units blood (range 0-5) per child a year was required to maintain the haemoglobin on dialysis. Episodes of septicaemia secondary to shunt or fistula infection were treated with parenteral cloxacillin and ampicillin in the first instance. 15 children had bilateral nephrectomies for persistent hypertension, with improvement in their condition and, often, better appetites and greater rates of growth. An average of one operation a year was required usually for shunt revision or fistula creation in a child on home dialysis.

One child developed pancreatitis due to overdosage of vitamin D but recovered after a period of dialysis in hospital. Three children underwent total parathyroidectomy for uncontrollable osteitis fibrosa cystica with hypercalcaemia on vitamin D supplements. No child required antihypertensive medication or digoxin. Convulsions were common early in the initial period on dialysis but were rare after they had returned home; only 2 children (with cystinosis) had a fit while on home dialysis. During the last 7 years no patient was or became Australia antigenpositive or developed hepatitis. Four boys on dialysis each had a priapism, 2 of whom needed surgical relief; neither can currently obtain an erection.

\section{Transplantation.}

\section{Patient survival (Fig. 7)}

46 of the 64 recipients who had transplants during the last 10 years are currently surviving with functioning allografts. 12 have died and 6 have returned to dialysis while awaiting a subsequent allograft, 3 after the 2 nd transplant had failed (Fig. 3).

Seven of the 12 deaths resulted from uraemia, generally with sepsis, after a firm decision had been taken not to offer further dialysis and retransplantation because a successful transplant was unlikely to be achieved, or because of the physical and emotional stress for the child and the family. One death occurred from pseudomembranous colitis, 1 from gastric haemorrhage after rejection, 1 from pancreatitis, 1 from a rhabdomyosarcoma, and 1 from electrolyte imbalance and cardiac arrest on peritoneal dialysis after graft failure.

\section{Graft survival}

$76 \%$ of live, related donor grafts, $46 \%$ of 1 st cadaver donor grafts, $39 \%$ of 2 nd cadaver donor grafts, and 1 of 3 rd cadaver donor grafts are still functioning 1 to 113 months after the transplant. Actuarial graft survivals for 1st grafts are shown in Fig. 8. Live donor graft survival was $65 \%$ at 3 years and $55 \%$ at 5 years. 1st cadaver graft survival was $42 \%$ at 5 years. Results obtained during the last 6 years of the 10-year period showed an improvement with live donor graft survival of $71 \%$ and a 1st cadaver graft survival of $47 \%$ at 3 years. $63 \%$ of cadaver donor grafts and all live donor grafts were matched for at least 2 HLA antigens, but no comment can be made about the effects of HLA matching as the groups are too small for analysis. The one live donor graft fully matched for HLA antigens was lost through rejection and 1 of 3 cadaver grafts fully matched was rejected. Crossmatching was negative for all grafts. The mixed lymphocyte cultures carried out on a number of patients showed little correlation with graft survival either for cadaver ${ }^{12}$ or live donor grafts.

\section{Rejection}

Acute rejection treatment was required at least once in all patients and up to 12 times in some in the first few months after transplantation. Good function was obtained eventually in some children who required up to 9 separate courses of treatment for rejection.

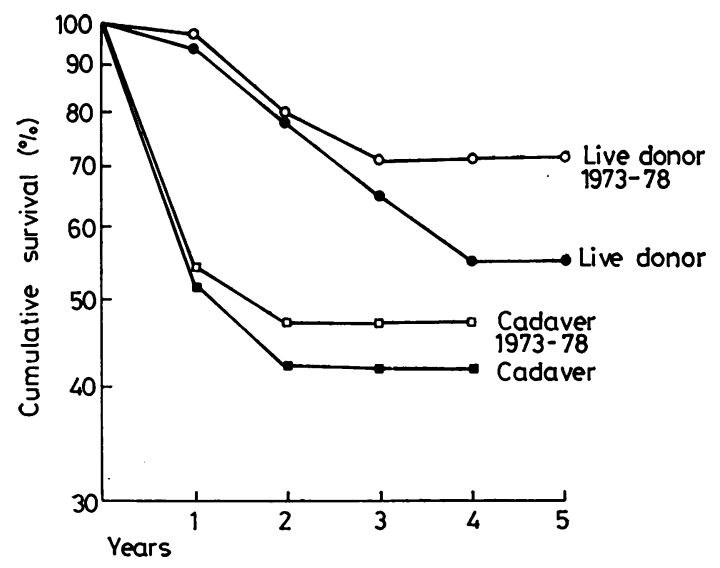

Fig. 8 Actuarial live donor and 1st cadaver graft survival. 


\section{Transplant failures}

Three patients were considered primary failures in that the graft never functioned and was removed. In one patient the renal vein of the donor kidney removed at another hospital was too short to effect an anastomosis so that the operation was not completed. In 16 cases, the graft was lost because of rejection, and the child returned to dialysis. In one case a pyonephrosis developed and one graft was removed because of failure to achieve adequate renal perfusion due to internal damage to the renal artery.

\section{Recurrence of original disease}

Recurrence of the original disease occurred in only 2 patients. Each had focal segmental glomerulosclerosis and developed massive proteinuria after transplantation. Diagnosis was confirmed on biopsy of the transplanted kidney; each has continued to function well up to 4 years after the transplant.

\section{Surgical complications}

The surgical complications encountered included haemorrhage/haematoma formation (7 patients), wound sepsis ( 5 patients), ureteric obstruction (4 patients), with ureteric leaks and fistula formation in 7 patients. Three patients had renal artery stenosis which required surgery and 3 had lymphoceles. One patient developed an iliac vein thrombosis in the late phase of transplantation and one patient had a small bowel obstruction.

\section{Other medical complications}

Anuria. 30 patients were anuric after the 76 successful transplantation operations and needed further periods (up to 5 weeks) on haemodialysis.

Hypertension. 21 patients developed hypertension postoperatively and required antihypertensive medications. Two patients developed hypertensive encephalopathy.

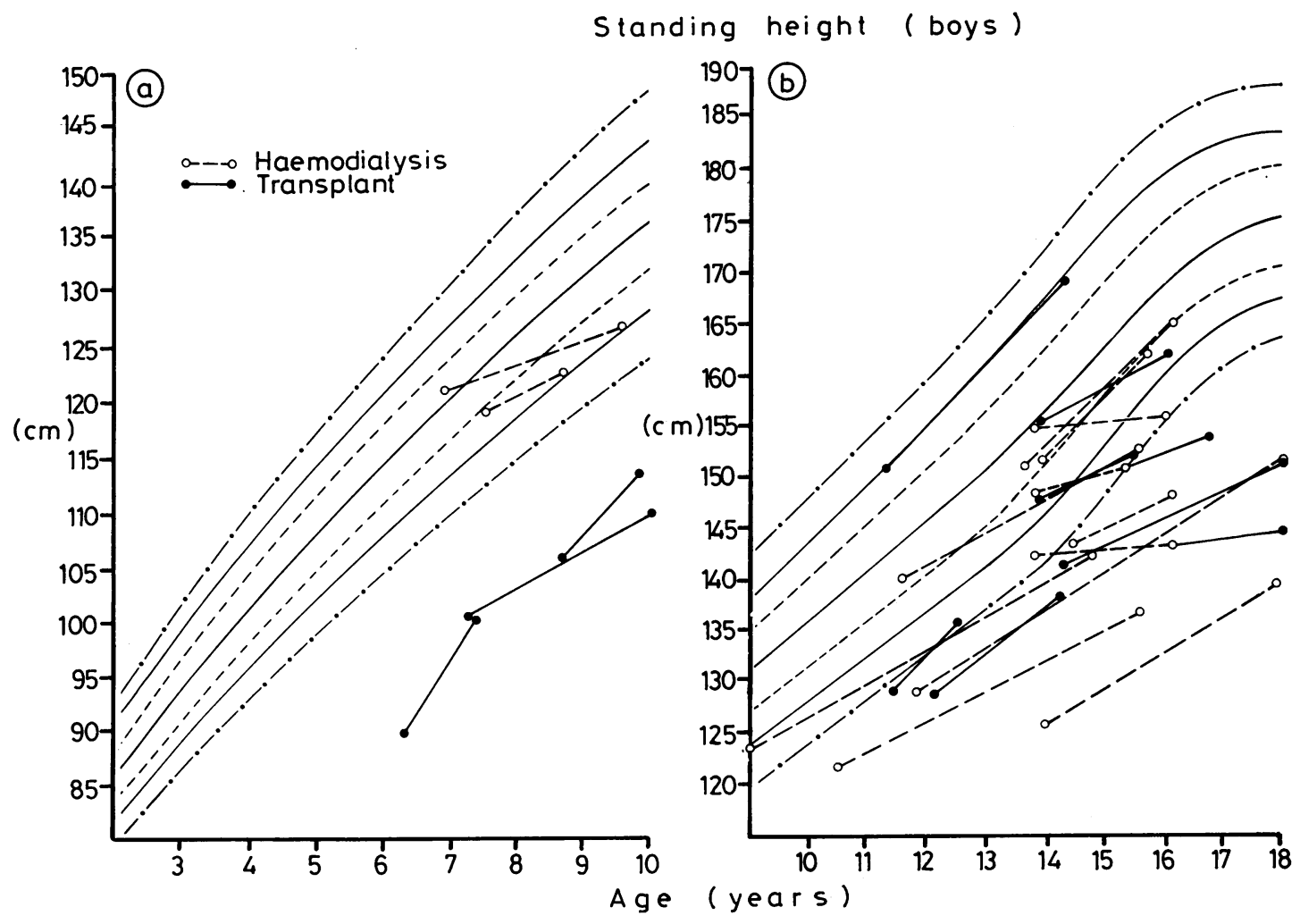

Fig. 9 Height centiles at beginning of treatment and at time of change of treatment, death, or in June 1978, for boys plotted against chronological age. Only children treated for more than 1 year are included. 
Urinary tract infection. 15 patients had 30 episodes of urinary tract infection caused by a variety of organisms, particularly Escherichia coli. Pseudomonas aeruginosa and Klebsiella aerobacter were also common infecting organisms.

Pyrexia of unknown origin. Three patients showed unremitting fever in the early postoperative phase for which no immediate cause could be found. Viral infection, particularly cytomegalovirus, was suspected in all and confirmed in one patient who recovered with full renal function in conjunction with a course of adenosine arabinoside.

Unusual infections. Generalised sepsis from Staphylococcus aureus was seen in 2 patients, viral myocarditis was encountered in 2 cases, and herpes zoster was seen in one patient. Two children had varicella infection treated with adenosine arabinoside but the attacks were slight, and the children survived. All children exposed to varicella were given hyperimmune gammaglobulin. Two patients early in the study became Australia antigen-positive during treatment and, when their kidneys failed, this became a factor in the decision for further treatment.

Corticosteroid toxicity. Weight gain and a cushingoid appearance were seen in all patients in the months immediately after transplantation but abated in the second year when all children were on alternate-day treatment with corticosteroids. Gross obesity remained a problem in some children, however. Steroid myopathy (3 patients), duodenal ulcer, and oesophagitis were also encountered. Three patients developed avascular necrosis of the hips.

Blood dyscrasia. Leucopenia ascribed to sensitivity to azathioprine and requiring adjustment of dosage occurred in 6 patients.

Hyperglycaemia. This was a problem in 3 patients with cystinosis in the postoperative phase and required short-term treatment with insulin.

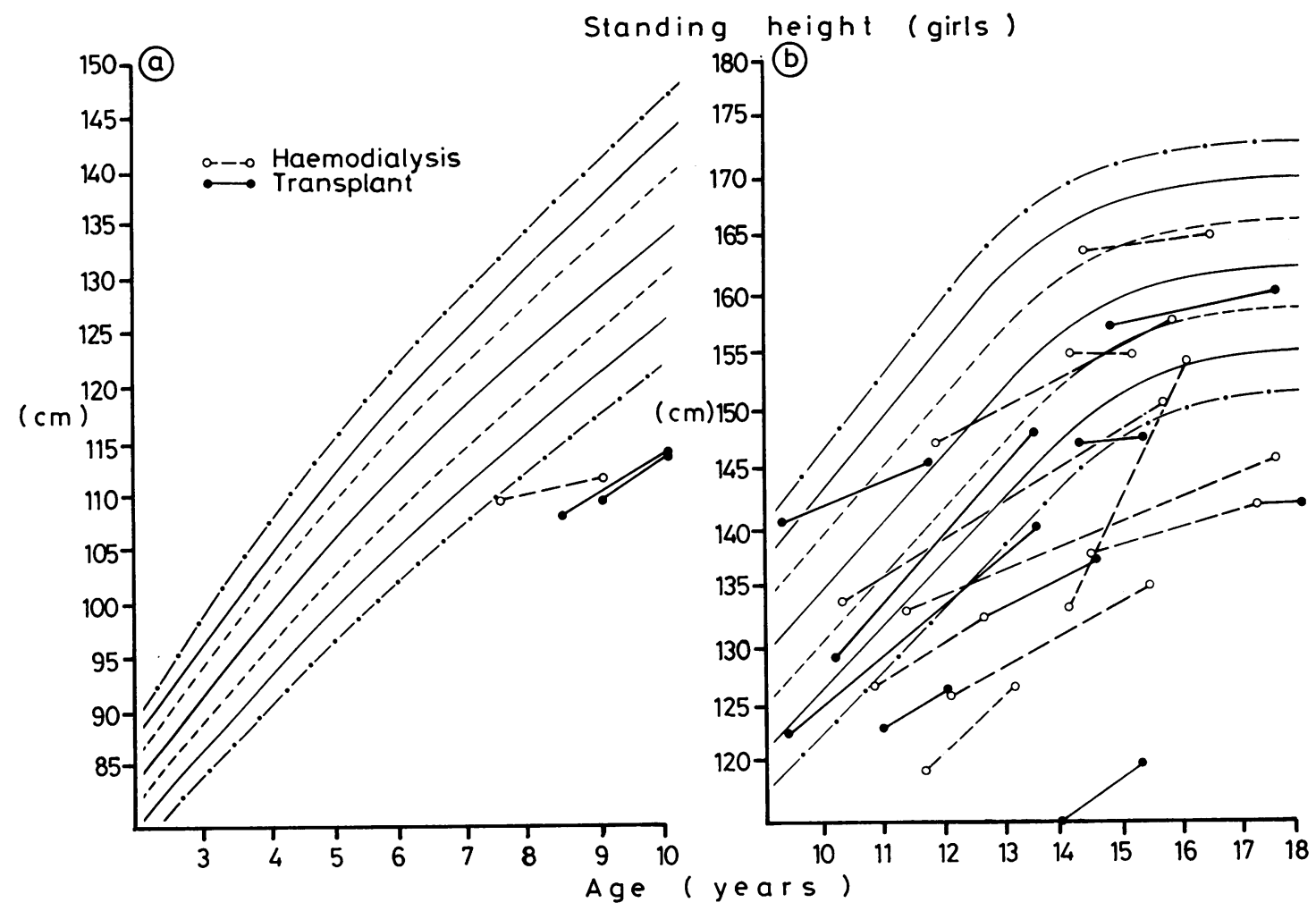

Fig. 10 Height centiles at beginning of treatment and at time of change of treatment, death, or in June 1978, for girls plotted against chronological age. Only children treated for more than 1 year are included. 
Convulsions. Six children each had at least one convulsion, generally in association with hypertension; of these, 2 children had cystinosis. Three continued to require anticonvulsive therapy even after the hypertension had been controlled.

\section{Growth}

Growth failure is a regular accompaniment of endstage renal failure. ${ }^{13} 40 \%$ of children entering this programme were below the 3rd centile for height for age. The overall growth of individual children followed for at least one year and plotted against chronological age is shown in Figs 9 and 10. Most children showed some growth although occasionally virtual growth arrest was seen. The growth patterns are analysed in Fig. 11. In prepubertal children (under 11 for boys and under 9 for girls), growth in boys after transplantation was better than for boys on haemodialysis. Growth was also better after transplantation in both girls and boys after puberty.

\section{Psychosocial problems}

Dealing with end-stage renal disease is complicated because most of the family is affected. Haemodialysis, particularly if conducted in the home, entails an unavoidable disruption in the family which may constitute either a cohesive or a disruptive force. A detailed psychosocial assessment of this problem has been published. ${ }^{5}$ Generally, a year on home dialysis was acceptable to about $80 \%$ of the children and to about $50 \%$ of the families. However, a successful transplant was regarded as being preferable and apart from anxieties about graft function and the tension during the transplant period there was resumption of normal family life. One adolescent girl failed to take the prescribed corticosteroids because of anxiety about her appearance after the

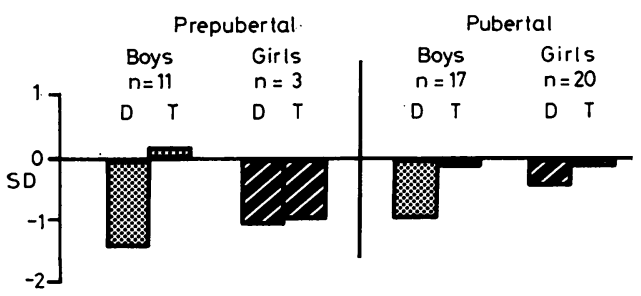

Fig. 11 Comparison of growth between dialysis $(D)$ and transplantation $(T)$ in children treated for at least 1 year. Pubertal status was defined as a chronological age of more than 11 years in boys and 9 years in girls. $S D$ is the standard deviation score calculated as the difference between the standard deviation of height from the mean of normal children of the same age at the start and at the end of the period of assessment. ${ }^{26}$
Table Number of children attending school after dialysis or transplant

\begin{tabular}{|c|c|c|c|c|c|c|}
\hline \multirow[t]{3}{*}{ School rehabilitation } & \multicolumn{4}{|c|}{ Dialysis } & \multirow{2}{*}{\multicolumn{2}{|c|}{$\frac{\text { Transplants }}{1 s t, 2 n d, \text { or } 3 r}$}} \\
\hline & \multicolumn{2}{|c|}{ Hospital } & \multicolumn{2}{|c|}{ Home } & & \\
\hline & No. & $\%$ & No. & $\%$ & No. & $\%$ \\
\hline Full time & & & 8 & 80 & 23 & 64 \\
\hline $\begin{array}{l}\text { Part time, with full } \\
\text { activity } \\
\text { Full time, with part }\end{array}$ & 1 & 25 & 1 & 10 & 3 & 8 \\
\hline $\begin{array}{l}\text { activity } \\
\text { Part time with part }\end{array}$ & & & & & 3 & 8 \\
\hline activity & 2 & 50 & 1 & 10 & 4 & 11 \\
\hline Unable to attend & 1 & 25 & & & 3 & 8 \\
\hline
\end{tabular}

transplantation and she is now back on dialysis. In none of the families has the stress led to a breakdown of the marriage.

50 of the children have been followed up to determine their rehabilitation as of June 1978. This is shown in the Table. $80 \%$ of children on home dialysis have full time school attendance or are very close to normal functioning. $64 \%$ of children who have had renal transplants are in this category. The percentage of children not functioning optimally comprise children currently on hospital haemodialysis as inpatients or children who have recently undergone renal transplantation and are in the process of being rehabilitated to full function.

\section{Discussion}

Regular dialysis and renal transplantation are accepted as effective methods for treating adults with terminal renal failure. Some centres have now reported their experience with paediatric dialysis and transplantation. ${ }^{14-16}$ The results are equally good. The figures from the European Dialysis Transplantation Association show that best results are obtained in children in the 10- to 14-year age group. ${ }^{117}$ Our results are comparable with other large series. The encouraging feature is the improved results obtained in the last 6 years. While there have been no major breakthroughs during this time, the results can be attributed to the intensive experience gained during the last 10 years, to the development of full paediatric facilities to handle the case load, to the close attention to medication including the use of alternate-day corticosteroids, ${ }^{18}$ to the earlier diagnosis of rejection with the help of renal scintigraphy ${ }^{11}$ and, perhaps, to the use of methylprednisolone pulse therapy for the treatment of acute rejection.

Our experience shows that a fully integrated service of dialysis, both peritoneal and haemodialysis, and renal transplantation are essential for success. Such a system allows the optimal mode of treatment to be chosen for a child at any time and allows him 
to move freely from one treatment to another. This series is unique because of the large number of children cared for by home haemodialysis. We have found this form of treatment to be suitable for many children while awaiting transplantation; the home conversion required is expensive although the new dialysis methods with regeneration of dialysis fluid require less plumbing and are portable, and unless a long period on dialysis is envisaged, home dialysis is often preferred. Increasingly the aim is for a short period on dialysis before transplantation.

Another unique feature is the high proportion of live related donor transplantations. There were 37 live related donors, most of them parents, in this series. No pressure was exerted on the family to offer the kidney. Before entering the chronic renal programme the various options were explained to the family, and the facts regarding transplantation and the results in this hospital were placed before them. Any decision regarding live related donors was made by the family; many families had been referred with the express intention of donating a kidney. Some donors were refused because of existing renal disease, often previously undiagnosed. Live related donor transplantation was discouraged if the family was considered unstable. Formal psychiatric assessment of the family was not however, a routine measure.

During the last 10 years the programme has been evaluated repeatedly and modifications made to the therapeutic regimen. Latterly our immunosuppressive protocol has been changed because we were concerned that sepsis was common. We were worried about the fairly high incidence of late rejection episodes and continuing loss of grafts at 2 and 3 years after the transplant, and we had seen some rare complications or associations related to our immunosuppressive therapy-for example, pancreatitis, rhabdyomyosarcoma, pseudomembranous colitis. Prednisolone dosage has been halved in the period after transplantation but increased to $30 \mathrm{mg} / \mathrm{m}^{2}$ every other day at one year. It is important not to risk the patient's life in a vain attempt to preserve graft function in a failing kidney by overenthusiastic immunosuppression.

The improved survival means that attention should be focused on quality of life. In paediatric practice therefore, optimal growth is a prime consideration. An unacceptably large number of children have severe growth retardation when they enter the chronic haemodialysis and renal transplantation programme and most of these patients continue to show a relatively low growth velocity while on dialysis or after transplantation; the poor growth in early life of children with chronic renal insufficiency is a major problem and an aggressive approach to management at this time is justified. ${ }^{13}$ Bone age is usually retarded in children with chronic renal failure and at least until after puberty height is often consistent with skeletal development. ${ }^{1}$ The growth data presented in this study are related to chronological age because adequate data on skeletal development were not collected early in the study and the final heights of these children may be significantly better than suggested by Figs 9 and 10. Many children showed useful and even catch-up growth although, overall, the height centiles deteriorated in all groups except for prepubertal boys after transplantation. When related to chronological age, growth was generally better after transplantation than on dialysis; the data accumulated by the European Dialysis and Transplantation Association show a similar trend. ${ }^{17}$ Sexual development is also delayed and obviously growth data should take account of this; unfortunately in the earlier patients we did not adequately document this so the separation shown in Fig. 11 has been taken at the mean age for onset of puberty in normal children. Finally in relation to growth, it should be stressed that good dietary advice for children on dialysis is important ${ }^{19-20}$ and that corticosteroid dosage after transplantation should be kept to a minimum.

Quality of life extends to the everyday activity of these children. The programme can be considered a success only if it exerts a minimal intrusion on their lives and they can be rehabilitated to as near normal life as possible. It has previously been documented that the school attendance of children on home haemodialysis is $65 \pm 15 \%$ : the national average for secondary schools is about $90 \%{ }^{5}$ Prolonged duration in hospital and close follow-up is required for at least 6 months after renal transplantation. After a year, apart from monthly visits to hospital and the necessity to remain on immunosuppressive agents, these children lead normal lives. The better growth and rehabilitation after transplantation make this, not dialysis, the aim of treatment; the shortage of donor kidneys for transplantation is critical and all doctors, including paediatricians, should be aware of the need.

The management of the child with end-stage renal failure and his family is complex. It involves dialysis machines, intricate surgery, and meticulous manipulation of dangerous drugs but in particular it involves a complex human element, a sympathetic understanding by the staff, the child, and his family. The development of full paediatric facilities with staff attuned to the varied needs of these children is an important aspect of the endeavour to provide a full and complete life to the child who presents with end-stage renal failure. ${ }^{21}$ Staff should include paediatric nephrologists, transplant surgeons, pae- 
diatric and dialysis nurses, hospital school teachers, specialised social workers and dietitians, and access to child psychiatry; facilities include paediatric wards, separate dialysis facilities for children, and ideally the close support of a university children's department and an adult nephrology department. ${ }^{22}$ The financial cost per patient is high ${ }^{23}$ but this is lower if the service is orientated to transplantation and is developed alongside an adult unit. Financial assistance with travelling costs is important for families and here we rely heavily on the British Kidney Patients Association for help.

Experience throughout Europe and in the USA suggests that while selection for treatment is desirable and that each child should be considered individually, some of the restrictions practised early in our study are no longer valid. Most doctors would still be doubtful about treating infants under age 2 years but results in children aged between 2 and 5 years are reasonable, ${ }^{1524}$ and a blanket restriction will probably not be tenable in the future.

About half the children in the UK with terminal renal failure die untreated ${ }^{25}$ because of lack of facilities. A plan for provision of care exists ${ }^{22}$ and has been accepted by the British Paediatric Association, the Renal Association, and the Department of Health, but has still not been fully implemented. Of the 12 units recommended for the treatment of children in terminal renal failure, only 5 are fully functional and the supraregional planning suggested in the BAPN report ${ }^{22}$ has not been undertaken. We suggest that the results are sufficiently good for it no longer to be reasonable to deny treatment to these children and therefore that the present public concern is justified.

We are grateful to the many doctors, nurses, physiotherapists, social workers, teachers, dietitians, and other staff who have cared for these children and their families. We are especially grateful for the support of the British Kidney Patient Association, National Kidney Research Fund, the Variety Club of Great Britain, and many other organisations and individuals who have contributed to the funding of the programme and the research connected with it. We gratefully acknowledge the support of the DHSS particularly with the study of home dialysis, and the continuing help of the regional and central medical officers, especially Dr G Pincherle.

\section{References}

1 Donckerwolcke R A, Chantler C, Brunner F P, et al. Combined report on dialysis and transplantation in Europe 1977. In: Robinson B H B, ed, Proceedings of the Fifteenth Congress of the European Dialysis and Transplantation Association. Tunbridge Wells: Pitman Medical, 1978: 77-116.
2 Riley C M. Thoughts about kidney homotransplantation in children. $J$ Pediatr 1964; 65: 797-800.

3 Ellis F, Berne T, Bewick M. The first renal transplant at Guy's Hospital. Guy's Hosp Rep 1972; 121 : 101-7.

- Boulton-Jones J M, Cameron J S, Bewick M, Ogg C S, Meadow S R, Ellis F G. Treatment of terminal renal failure in children by home dialysis and transplantation. Arch Dis Child 1971; 46: 457-63.

5 Wass V J, Barratt T M, Howarth R V, et al. Home haemodialysis in children. Report of the London Children's Home Dialysis Group. Lancet 1977; i: 242-6.

- Bewick M. Haemodialysis and transplantation. In: Eckstein H B, Hohenfellner R, Williams D I, eds. Surgical paediatric urology. Stuttgart: Thieme, 1977: 503-17.

7 Scharer K, Chantler C, Donckerwolcke R. Paediatric dialysis. In: Drukker W, Parsons F M, Maher J F, eds. Replacement of renal function by dialysis. The Hague: Martinus Nijhoff Medical Division, 1978; 444-61.

8 Botella J, Traver J A, Sanz Guajardo D, Torres M T, Sanjuan I, Zabala P. Chloramines, an aggravating factor in the anaemia of patients on regular dialysis treatment. In: Robinson B H B, ed. Dialysis, transplantation, nephrology. Proceedings of the Fourteenth Congress of the European Dialysis and Transplantation Association. Tunbridge Wells: Pitman Medical, 1977: 192-9.

- El-Bishti M, Counahan R, Jarrett R J, Stimmler L, Wass $\mathrm{V}$, Chantler C. Hyperlipidaemia in children on regular haemodialysis. Arch Dis Child 1977; 52: 932-6.

10 Chantler C, El-Bishti M, Counahan R, Wass V J, Cox B D. Growth in children with renal failure. Med Mitt (Melsungen) 1976; 50: Supplement 11, 57-70.

11 Burke J R, Counahan R, Hilson A J W, et al. Serial quantitative imaging with 99 TCmDTPA in paediatric renal transplantation. Clin Nephrol 1979; 12: 174-7.

12 Jones A R, Bewick M, Vaughan R W, Batchelor J R. Transformation of lymphocytes from patients awaiting cadaver renal transplants. Lancet 1976; ii: 529-31.

13 Holliday M A, ed. Metabolism and growth in children with kidney insufficiency. Kidney Int 1978; 14: 299-382.

14 Hulme D, Kenyon J R, Owen K, et al. Renal transplantation in children. Arch Dis Child 1972; 47: 486-94.

15 Hodson E M, Najarian J S, Kjellstrand C M, Simmons R L, Mauer E M. Renal transplantation in children ages 1 to 5 years. Pediatrics 1978; 61: 458-64.

16 Fine R N, Malezadeh M H, Pennisi A J, et al. Long term results of renal transplantation in children. Pediatrics 1978 ; 61: 641-50.

17 Chantler C, Donckerwolcke R A, Brunner F P, et al. Combined report on regular dialysis and transplantation of children in Europe 1976. In: Robinson B H B, ed. Dialysis, transplantation, nephrology. Proceedings of the Fourteenth Congress of the European Dialysis and Transplantation Association. Tunbridge Wells: Pitman Medical, 1977: 70-112.

18 Reimold E W. Intermittent prednisolone therapy in children and adolescents after renal transplantation. Pediatrics 1973; 52: 235-40.

19 Chantler C, Holliday M A. Growth in children with renal disease, with particular reference to the effects of calorie malnutrition. Clin Nephrol 1973; 1 : 230-42.

20 Chantler C, Holliday M A. Metabolism and nutritional factors in children with renal insufficiency. Kidney Int 1978; 14: 306-12.

21 Cameron J S. The treatment of chronic renal failure in children by regular dialysis and by transplantation. Nephron 1973; 11: 221-51. 
22 Houston I, ed. Report: Siting of units to care for children with chronic renal failure in the United Kingdom: a progress report 1974-79. British Association of Paediatric Nephrology, Professor I Houston, Royal Manchester Children's Hospital, Pendlebury, Manchester M27 1HA, 1980: in press.

23 Office of Health Economics. Renal failure. A priority in health? Report No. 62. London: OHE, 1978.

24 Broyer M. Survey of the experience of the French paediatric units with dialysis and transplant programmes in children under 5 years of age (abstract). Arch Dis Child 1979; 54: 321.
25 Chantler C, Barratt T M. Letter: Priorities and childhood renal failure. Lancet 1976; i: 583.

26 Potter D E, Broyer M, Chantler C, et al. Measurement of growth in children with renal insufficiency. Kidney Int 1978; 14: 378-82.

Correspondence to Dr C Chantler, Evelina Children's Department, Guy's Hospital, St Thomas Street, London SE1 9RT.

Received 26 June 1979 